\title{
Evaluation of the Tuberculosis Surveillance System in Shamva District, Zimbabwe, 2014
}

Richard Makurumidze ${ }^{1}$, Notion Gombe ${ }^{1}$, Donewell Bangure ${ }^{1}$, Lucia Takundwa ${ }^{1}$, Cremance Tshuma ${ }^{2}$, Tapiwa Magure $^{3}$ and Mufuta Tshimanga ${ }^{1}$

1. Department of Community Medicine, University of Zimbabwe College of Health Sciences, Harare +263(0)4, Zimbabwe

2. Provincial Medical Directorate, Mashonaland Central Province, Bindura +263(0)71, Zimbabwe

3. National AIDS Council of Zimbabwe, Harare +263(0)4, Zimbabwe

\begin{abstract}
A review of 2013 tuberculosis (TB) surveillance data for Shamva District showed shortcomings. An average of 10 health facilities out 15 were reporting TB data on time. There was incomplete and incorrect filling of TB surveillance tools. A mixed methods (quantitative and qualitative) study was conducted between January and June 2014 and the study participants were healthcare workers. Data was collected using questionnaires and interview guide for key informants. Quantitative data was analysed using Epi-Info 7. Forty one (41) health care workers were interviewed. Females constituted (70.3\%) and males 29.3\%. Median age in years was 39 (IQR: 33-45) and median length of service in years was 6 (IQR: 4-9). The majority of the healthcare workers were Primary Care Nurses (43.9\%). Only $22 \%$ of health care workers were trained in TB disease surveillance. Knowledge on TB symptoms and signs was above $90 \%$ but between $60 \%-90 \%$ on the objectives of the surveillance system. Lack of transport; lack of stationery; delay in compilation; negative attitude and motivation were mentioned as reasons for delays in reporting. Thirty three $(80.5 \%)$ mentioned that the TB data collected is used in any way at their health facility. Only $19.5 \%$ had access to TB screening flow chart. $85 \%$ of the healthcare workers were willing to complete TB notification forms. Majority of health facilities did not have computers, internet access, faxes and landlines. Shortage of various TB surveillance tools was reported. We therefore concluded that knowledge levels were poor on the objectives of the surveillance system. The TB surveillance system was not simple, stable and timely but was useful, flexible, and acceptable. Mentoring, training and capacity building of healthcare workers in TB surveillance and provision of surveillance tools will improve the system performance.
\end{abstract}

Key words: Tuberculosis, surveillance system, Zimbabwe.

\section{Introduction}

Public health surveillance is defined as the "ongoing systematic collection, analysis, and interpretation of data essential to the planning, implementation, and evaluation of public health practice, closely integrated with the timely dissemination of these data to those who need to know" [1]. Such surveillance can serve as an early warning system for impending public health emergencies. It documents the impact of an intervention, or tracks progress towards specified goals and monitors/clarifies the epidemiology of health problems, to allow priorities to be set and to inform

Corresponding Author: Richard Makurumidze MPH, public health physician, research field: HIV, tuberculosis and public health. public health policy and strategies [2]. The core functions in surveillance of any health event include case detection, reporting, investigation and confirmation, analysis, interpretation, taking action in the form of control/response, formulating policy and feedback [3]. Reasons for conducting public health surveillance can include the need to assess the health status of a population, establish public health priorities, and reduce the burden of disease in a population by appropriately targeting effective disease prevention and control activities [1]. However, distinction should be made between surveillance and mere data collection the major difference being that with surveillance "action" is taken [2].

Tuberculosis (TB) is the second greatest cause of 
death worldwide due to a single infectious agent after HIV \& AIDS. In 2011 alone, nearly 9 million people around the world developed TB disease resulting in around 2 million TB-related deaths. Sub-Saharan Africa had the highest incidence in 2010 at over 270 cases per 100000 people with $80 \%$ of reported TB cases occurring in only 22 countries [4]. Zimbabwe ranks 17 out of these 22 highly burdened TB countries. TB is one of the top five leading causes of Out Patients Department (OPD) consultations and admissions in Zimbabwe [5]. It is the second leading cause of death for all inpatients [4]. Tuberculosis surveillance is a critical component for successful tuberculosis control, providing essential information needed to determine tuberculosis patterns and trends; identify high-risk populations and settings, multidrug resistance disease and establish priorities for control and prevention activities for the disease. The objectives of the Zimbabwe National TB Control Program are to: (1) detect at least $70 \%$ of new infectious TB cases; (2) successfully treat at least $85 \%$ of new infectious TB cases; (3) reduce the prevalence of and death due to TB and so reduce the social and economic burden placed by TB upon families and communities and (4) eliminate TB as a public health problem in Zimbabwe [5].

In Shamva District, TB surveillance is coordinated by the District TB Coordinator who is based at Shamva District Hospital. The 15 public rural health centres in the district are supposed to report TB data every month to the district. Each health facility is supposed to analyse its TB data and recommendations given to take appropriate actions before the data is forwarded to the next level which is the district.

A review of 2013 tuberculosis surveillance data and an interview with the Shamva District Tuberculosis (TB) Coordinator revealed shortcomings of tuberculosis surveillance system in the district. The Direct Observed Therapy (DOTS) Register at Shamva District Hospital was blank despite the hospital having 15 patients registered to collect drugs at the institution.
The laboratory request forms were left blank or incorrectly completed on the reason for sputum collection. Some laboratory request forms did not show whether the sputum was the initial, end of intensive phase or end of continuation phase. Notification forms were also incompletely filled, the common mistake being incorrectly ticking on sputum negative tuberculosis for tuberculosis diagnosed based on chest X-Ray. The majority of rural addresses were not specific and clearly identifying the village and headmen in all the registers and forms which made follow up difficult. An average of 10 health facility out of the 15 submitted monthly report on time to District TB Coordinator during 2013. Out of the expected 4 Quarterly TB Review Meeting during which data is shared, analysed and discussed; the district only managed to conduct one meeting in 2013. We therefore evaluated the tuberculosis surveillance system in Shamva District.

\section{Materials and Methods}

The study setting was Shamva District. The district is one of the 6 districts in Mashonaland Central Province in Zimbabwe. The district is about $90 \mathrm{~km}$ from Harare, the capital city of Zimbabwe. It has a population of 109,572 according to the 2010 Zimbabwe Statistical Office census data. The district main economic activity is mining with Shamva Gold Mine being the largest mine in the district. The district is serviced by 15 health centres which include two hospitals (Shamva District Hospital and Madziwa Rural Hospital) and 13 Rural Health Centres.

A mixed methods (quantitative and qualitative) study was conducted. The study population were health workers in the district. Health care worker who were on duty during the days of the data collection period were recruited into the study. The District TB Coordinator, District Medical Officer and District Health Information Officer were purposively included as key informants. All the records in the Presumptive TB Register for 2013 were used to assess data quality. 
Data was collected using an interviewer administered questionnaire and an interview guide for key informants. The questionnaire was designed in line with Centre for Disease Control Guidelines on Surveillance of a Public Health System. Checklists were used to assess the surveillance system attributes (data quality, timeliness and stability). A pre-test of data collecting tools i.e. the questionnaire, interview guide and checklist was conducted prior to commencement of the study. The data collection tools were then adjusted according to appropriateness, timing, interpretation, sequence and wording of questions.

Quantitative data was analysed using Epi Info 7. Frequencies of various variables were calculated and data was then summarised using tables, graphs and numerically using measures of central tendency and dispersion. Qualitative data was analysed thematically. The permission to carry out the study was sought from the Mashonaland Central Provincial Medical Director and the Shamva District Medical Officer. The study ethical considerations were assessed and approved by the Health Studies Office within the Ministry of Health and Child Care. A written consent form was obtained from every participant prior to entry into the study. No names were written on the data collection tools. Confidentiality was assured and maintained.

\section{Findings}

Ten (10) health facilities were visited in Shamva District and from them 41 participants were interviewed. The majority of the participants were females $(70.3 \%$ ) and males constituted $29.3 \%$. The median age in years for the study participants was 39 (IQR: 33-45). The median length in service in years for the study participants was 6 (IQR: 4-9) (Table 1).

The health care workers knowledge on the surveillance system was assessed. Only $22 \%$ of the health care workers were trained in TB disease surveillance. Knowledge on TB symptoms and signs was above $90 \%$. None of the health care workers had an idea on how to calculate health facility TB threshold. The knowledge parameters assessed are summarised in Table 2 .

Table 1 Characteristics of study participants.

\begin{tabular}{|c|c|c|}
\hline Variable & Category & Frequency $n=41(\%)$ \\
\hline \multirow{2}{*}{ Sex } & Female & $29(70.7)$ \\
\hline & Male & $12(29.3)$ \\
\hline \multirow{6}{*}{ Profession } & Primary Care Nurses & $18(43.9)$ \\
\hline & Registered General Nurses & $15(36.6)$ \\
\hline & Environmental Heath Technician/Officer & $4(9.8)$ \\
\hline & State Certified Nurses & $2(4.9)$ \\
\hline & Health Information Officer & $1(2.4)$ \\
\hline & Microscopist & $1(2.4)$ \\
\hline
\end{tabular}

Table 2 Health care worker knowledge of the surveillance system.

\begin{tabular}{lll}
\hline Variable & Category & Frequency $n=41(\%)$ \\
\hline Training in TB Surveillance & Yes & $9(22.0)$ \\
TB Detection & Two sputum collected for DSM & $39(95.1)$ \\
& DSM standard for TB Diagnosis & $33(80.5)$ \\
TB Symptoms and Signs & Weight loss & $40(97.6)$ \\
& Fever & $39(95.1)$ \\
& Night sweats & $40(97.6)$ \\
Objectives on TB surveillance & Cough for 2 weeks & $38(92.7)$ \\
& Detect 70\% of cases & $28(68.3)$ \\
Calculation of TB Thresholds & Treat 85\% of all cases & $33(80.5)$ \\
& Reduce prevalence and death & $40(97.6)$ \\
& Eliminate TB as public health problem & $37(90.2)$ \\
\hline
\end{tabular}


Twenty one 21 (51.2\%) of the participants mentioned that they participated in the compilation of the TB monthly report. The participants were asked where the TB monthly report is submitted and 26 (63.4\%) knew that it was to the District Health Information Office. The participants were asked on the mode of transport they use to submit the TB monthly report and the response were as follows: collected by district office $2 / 41$; health facility transport $8 / 41$; public transport $28 / 41$ and other $3 / 41$. Out the 28 which mentioned that they use public transport $13 / 28$ of them said they were provided with bus fare. The study participants were asked to mention the reason for delaying to report TB surveillance data; the majority mentioned lack of transport followed by delay in compilation; negative attitude and motivation then lack of stationery. The response are summarised in Fig. 1.

The study participants were asked on reporting deadlines. The majority of the participants were unaware of the reporting deadlines. Only $7.3 \%$ knew the compilation deadline for health facility report and
$19.5 \%$ knew the due date for submission to the district.

\subsection{Usefulness}

Thirty three $(80.5 \%)$ mentioned that the TB data collected is used in any way at their health facility. The mentioned uses are summarised in Fig. 2. The most mentioned use was planning followed by monitoring \& evaluation, outbreak detection and research. Outbreak detection was mentioned but no TB outbreak was detected in the area in the previous year. Fifty one percent $(51.0 \%)$ of the health care workers mentioned that they review TB data locally; $31.7 \%$ mentioned that TB reports are circulated; $26.8 \%$ were able to assess trends and outbreaks from TB reports and $43.9 \%$ mentioned that they attend TB review meetings.

\subsection{Data Quality}

448 TB records (entries) in the Presumptive TB Register were used to assess data quality. 311 out of 448 (69.5\%) had all fields filled $100 \%$ completeness. $34 \%$ of all entries assessed did not have full physical

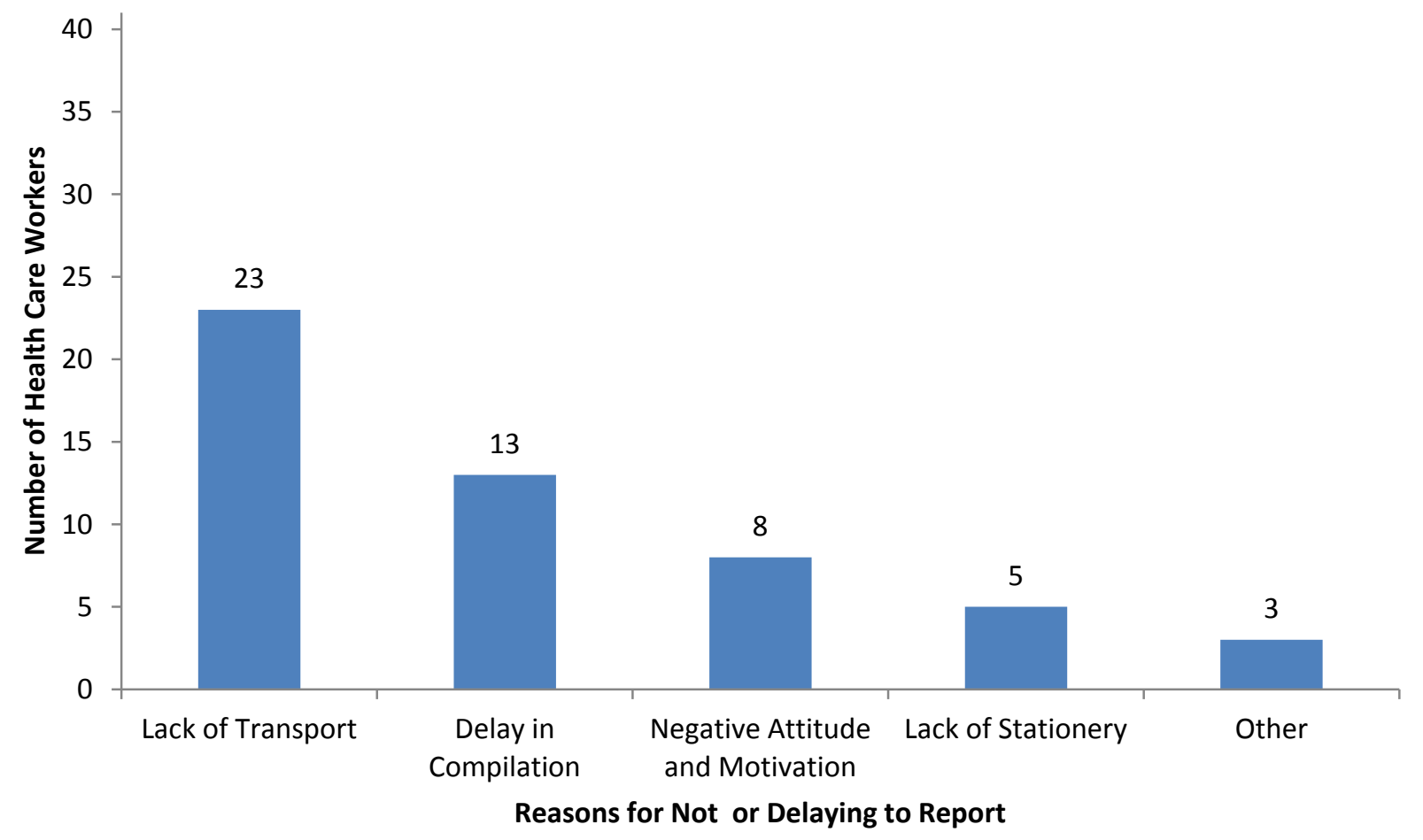

Fig. 1 Reasons for delaying or not reporting TB data. 


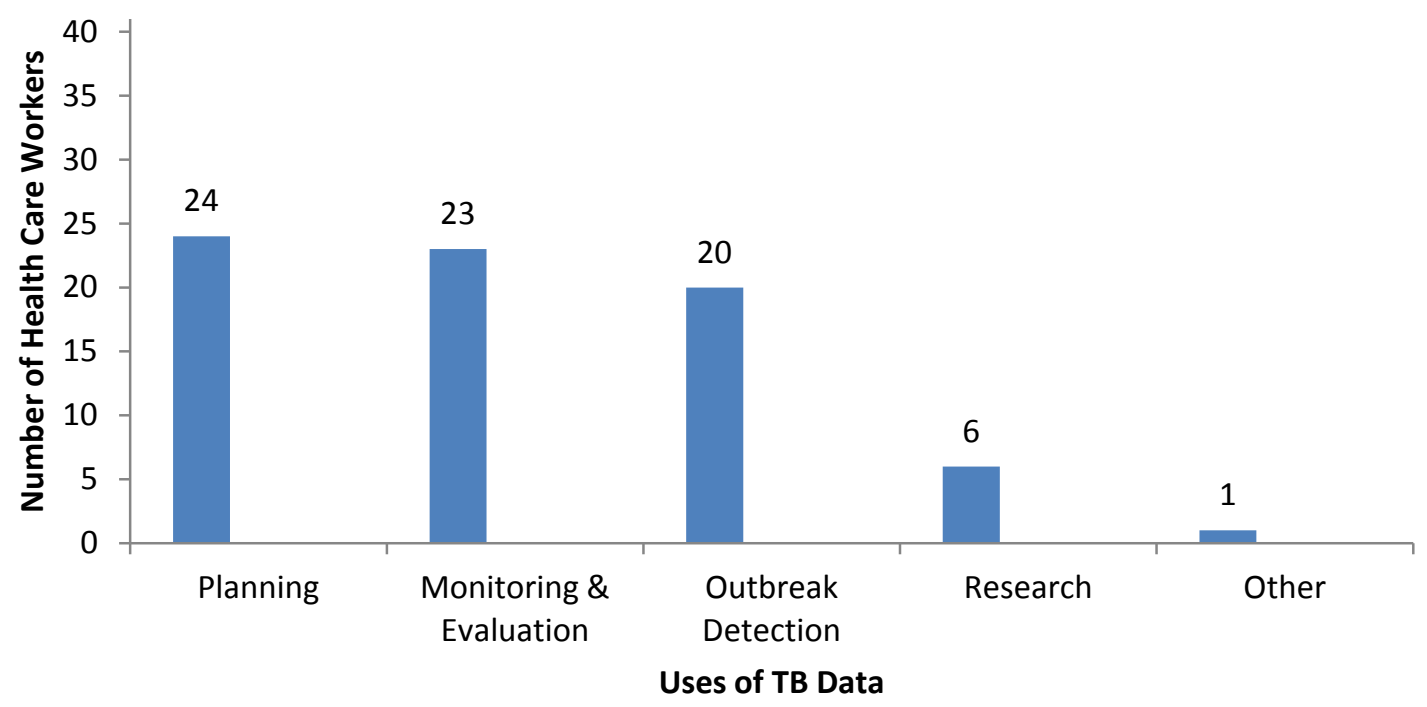

Fig. 2 Uses of TB surveillance data in Shamva district.

address of the TB presumptive cases, $57 \%$ did not have second sputum tests done, $24 \%$ did not have TB presumptive case number and $12 \%$ did not indicate sex of the clients. 372 out of 448 (83\%) entries assessed had data consistent with data in the laboratory register (validity). 442 (98.7\%) entries were legible. The TB Quarterly Summary Notification Forms (4) for 2013 were $100 \%$ completed.

\subsection{Simplicity}

The health care workers were asked if they had access to a TB screening flow chart and only $19.5 \%$ had access. The majority (63.4\%) of them mentioned that they needed training in TB surveillance and only $41.5 \%$ (17/41) once completed the TB notification forms. Those who once completed at TB notification the majority (12/17) of them took 4-6 minutes to complete the form; mentioned that the information required is enough (15/17) and (17/17) mentioned that there were no difficult sections on the form.

\subsection{Flexibility}

There was integration of HIV \& AIDS and TB activities in the district. The surveillance system was linked to other systems which included the PMTCT and $\mathrm{T}$ series. Changes in the TB surveillance tools were mentioned which included duration of cough for HIV patients on screening tool, TB suspect register to TB presumptive register and inclusion of MDR.

\subsection{Acceptability}

The health care workers were asked to mention who had the responsibility of completing TB notification forms at their health facilities. $63 \%$ mentioned that it was the responsibility of the nurses or doctors, $27 \%$ mentioned Environmental Health Technician/Officer and $10 \%$ Health Information Officers. $85 \%$ of the health care workers were willing to complete TB notification forms and $88 \%$ felt it is part of their duties to fill TB notification forms.

\subsection{Timeliness}

The health care workers were asked the time it takes for the sputum to reach the laboratory from their health facility. The median time in days for the sample to reach the laboratory was 1 (IQR: 1-2) and $29.3 \%$ of the health workers thought there was a delay in the process.

The health care workers were asked the time it takes for the results to reach the health facility back from the laboratory. The median time in days it took the results to reach the health facility was 3 (IQR: 2-7) and 36.6\% 
Table 3 Availability of TB surveillance tools at health facility level.

\begin{tabular}{ll}
\hline Surveillance Tool & Health Facilities with Tool Available $n=10$ \\
\hline TB Guidelines & 8 \\
Sputum Request Forms & 7 \\
TB DOT Register & 10 \\
TB Notification Forms & 5 \\
TB Screening Tool Flow Chart & 5 \\
TB Suspect Register & 10 \\
TB Treatment Cards & 5 \\
Transfer Patient Report Form & 10 \\
TB Laboratory Register & 2 \\
\hline
\end{tabular}

Table 4 Availability of TB surveillance tools at district level.

\begin{tabular}{ll}
\hline Item & Availability \\
\hline List of all TB Health facilities & Yes \\
Most Recent Annual TB report & No \\
TB Quarterly Summary Notification Forms & Yes \\
TB Treatment Outcome Request Form & No \\
TB Treatment Outcome Report Form & No \\
TB Quarterly Report Form on Treatment Outcome and TB/HIV Activities & Yes \\
Reports on data quality or surveillance evaluations in the last 5 years & No \\
\hline
\end{tabular}

of the health workers thought there was a delay in the process.

The health care workers were asked the time it took the health facility to submit the monthly TB report to the district after compilation. The median time in days it took the report to reach the district was 8 (IQR: 3-12) and $56.1 \%$ of the health workers thought there was a delay in the process.

\subsection{Stability}

All the health facilities had cell phones for the weekly disease surveillance system. The majority of the health facilities did not have computers, internet access, landlines, functioning faxes and radios. All the health facilities visited had TB DOT Registers, TB Presumptive Registers and Transfer Patient Report Forms. TB Laboratory Registers were only available at the two diagnostic centres in the district. There was shortage of other various TB surveillance tools and this is summarised in Table 3 .

At the district level, the 2013 Annual TB Report was not yet available. TB Treatment Outcome Request and Report Forms were not available. There were also no reports of data quality or surveillance evaluation in the last five years. The other surveillance tools assessed are shown in Table 4.

\section{Discussion}

Knowledge on the objectives of the surveillance was poor among the study participants though it was good on signs and symptoms of TB. Poor knowledge of the TB surveillance system was also found in similar evaluations done locally [6, 7]. The low knowledge level on the surveillance system could be explained by the fact only $22 \%$ of the health workers were trained in TB disease surveillance. In the delivery of health services, training is crucial in understanding practical components. The other reason why knowledge was poor in the study might be due to fact that the majority of our study participants were Primary Care Nurses. The training of primary care nurses is not very intensive and usually they are not given opportunities to attend workshops and trainings due to their junior positions. None of the study participants was able to calculate TB thresholds and this could have been explained by the fact that currently; the national the 
number of TB cases is going down with the advent of antiretroviral therapy reducing the likeliness of having of TB outbreak and policy makers are reluctant to discuss such issues in capacity building platforms.

Our study showed poor knowledge of the reporting deadlines by the health care workers and the finding is consistent to a study by Choto et al. in 2010 [6]. The completeness of data was also very low $(69.5 \%)$ with a lot of important data being incomplete in the TB Presumptive Register. The findings are however consistent with studies done in the United States using various combinations of data i.e. laboratory, clinic, pharmacy, and hospital discharge records which showed completeness of TB data ranging from $65 \%$ to 99.5\% [8]. Poor data quality makes information unreliable. This poor knowledge of reporting deadlines and data quality could also be explained by fact that very few health workers interviewed were trained in disease surveillance.

About seventy one percent $(70.7 \%)$ of the health workers were females. This findings show that the health profession mainly the nursing field is stills a female dominated profession since the majority of our study participants were nurses. Our low median number of years in service could be indicative of a high staff turnover and this might also explain the poor knowledge of the surveillance system.

Our study results showed timeliness on the submission of sputum and receiving of results back from the laboratory. This could be explained by the fact that the district is a beneficiary of the Ministry of Health and Child Care "Sample Run" programme which is supported by donors to promote quick transportation of samples to processing centres. The samples under the Sample Run programme include TB sputum for AAFBS and Dried Blood Spot for diagnoses of HIV infection in children. However, the system was not timely on submission of reports since report collection is not integrated into the Sample Run programme. Long reporting lags limit the usefulness of data for TB surveillance. Complete and timely notification of TB to public health authorities is one of the essential components of TB control. Notification of TB initiates assessment of TB cases, assignment of directly observed therapy (DOT) observers and contact tracing. Timely notification of TB provides surveillance data to keep track of the epidemic of tuberculosis.

According to our study, the surveillance system was not simple to implement due to several reasons. The majority of the health care workers had no access to the TB screening tool which helps and guides the screening process. A significant number of the health care worker mentioned that they needed additional training in various aspects of the surveillance including completion of the TB notification form because less than half of them had once completed the TB notification form. Our findings are however contrast to other evaluations of TB surveillance systems which showed the system to be simpler due to availability of flow charts [7, 9].

The system was flexible because in our study a significant number of health care workers mentioned integration of HIV \& AIDS and TB activities, use of additional information, use of information technology, recent changes in TB surveillance tools and ability to add extra information. The findings are also consistent with other similar evaluations done locally $[6,9,10]$. The flexibility of the system can however be improved by improving use of information technology and implementing newer technology like Gene Expert.

According to findings of our study the system was not stable. Majority of the health institution did not have access to internet and computers; functioning landlines, fax and radios. The median age in service for the health workers was also low. The finding is however contrasted to a finding by Gaka et al. which showed stable TB surveillance system [7]. The reason why the system is unstable might be due to the economic meltdown the country is facing which has led to the deterioration of infrastructure and high turnover of staff. 


\section{Conclusion}

Knowledge on the TB surveillance was poor mainly on the objectives of the surveillance system. This was largely a result of lack of training on disease surveillance among the health care workers. The TB surveillance system was not simple and not stable but was flexible and acceptable. The surveillance system was found to be useful. The information was used for planning, outbreak detection and monitoring and evaluation of the programme. Data quality was poor as shown by low levels of completeness and validity. Submission of sputum specimens and receiving results back from the laboratory was timely but submission of TB monthly report was untimely due to lack of transport and poor knowledge of reporting deadlines. There was shortage of TB surveillance tools at some health facilities in the district.

We therefore recommended completion of TB Missing data in the TB Presumptive Registers for easy follow up; use the Sample Run motorbikes to collect monthly reports; mentoring, training and capacity building of health care workers in TB surveillance; use of information technology and regular supply of TB surveillance tools to health facilities to improve the surveillance system.

\section{Acknowledgments}

We would like to acknowledge the following for the support we received during our study. Ministry of Health and Child Care, Health Studies Office; University of Zimbabwe Department of Community Medicine; National AIDS Council of Zimbabwe;
Centre for Disease Control Zimbabwe; Mashonaland Central Province Medical Directorate and Shamva District Medical Office

\section{References}

[1] Thacker, S. B., and Berkelman, R. L. 1988. "Public Health Surveillance in the United States." Epidemiol. Rev. 10: 164-90.

[2] German, R. R., Lee, L. M., Horan, J. M., Milstein, R. L., Pertowski, C. A., and Waller, M. N. 2001. "Updated Guidelines for Evaluating Public Health Surveillance Systems: Recommendations from the Guidelines Working Group." MMWR. Recomm. Rep. 50 (RR-13): 1-35.

[3] World Health Organization. 2000. Recommended Surveillance Standards, 2nd Edition. Geneva, Switzerland. Accessed May 2016. http://www.who.int/csr/resources/publications/surveillanc e/WHO_CDS_CSR_ISR_99_2_EN/en/

[4] World Health Organization. 2012. Global Tuberculosis Control 2011 Report. Geneva, Switzerland.

[5] Zimbabwe National AIDS and TB Program. 2010. National Tuberculosis Guidelines, 4th Edition.

[6] Choto, R. C., Midzi, S. M., and Mberikunashe, J. 2010. "Evaluation of the Prison Weekly Disease Surveillance System in Harare Province." Zimbabwe Field Epidemiology Training Program.

[7] Gaka, E., and Magure, T. 2013. "Evaluation of the Tuberculosis Surveillance Systems in Prisons, Harare." Zimbabwe Field Epidemiology Training Program.

[8] Murray, R. J., Hayden, C. H., and Zahn, F. 1974. "Irregular Reporting of Tuberculosis Cases by Laboratories in Nassau County." Public Health Rep. 89 (4): 385-8.

[9] Magure, T., and Nkomo, I. 1999. "Evaluation of TB Notifications in Bulawayo City, Zimbabwe.” Zimbabwe Field Epidemiology Training Program.

[10] Muchedzi, A., and Dhliwayo, P. 2006. "Tuberculosis Surveillance in the prisons in Harare, Zimbabwe." Zimbabwe Field Epidemiology Training Program. 